\title{
Los relieves del Potnios Hippōn de Lorca (Murcia)
}

\author{
JORGE JUAN EIROA *
}

Recientemente hemos dado a conocer la existencia de dos nuevas representaciones del "domador de caballos", halladas en Lorca (Murcia) hace 50 años, en el paraje denominado Hoya de la Escarihuela, en un bancal al que se llama "del tesoro" en el que, además, han aparecido en recientes prospecciones (1988) materiales arqueológicos cuyo inventario ya se ha pormemorizado (Eiroa y Martínez, 1988, 127), entre los que destacaremos la presencia de escasos fragmentos de cerámica ibérica que pueden fecharse entre los siglos III-II a. J.C., así como otros de sigillata aretina, paredes finas y un número mayor de fragmentos de sigillata sudgálica y Clara $A$ y algunos de Clara $D$, además de un ánfora africana casi completa del tipo VII de Keay (Keay, 1984, 123) de la primera mitad del siglo $V$ d. J.C.; de forma que el enclave parece haber tenido una larga ocupación, con una fase de apogeo entre los siglos I y mediados del II d. J.C., prolongándose hasta época tardoantigua.

Los relieves, que permanecieron ocultos hasta ahora, se conservan hoy en el Centro Municipal de Arqueología de Lorca, a la espera de ser instalados en el futuro Museo local, tienen las siguientes caracteristicas (lám. I y fig. 1):

RELIEVE 1. Está esculpido sobre un bloque de piedra organógenea, de $40 \mathrm{~cm}$. de altura, $31 \mathrm{~cm}$. de anchura y $22 \mathrm{~cm}$. de fondo, al que le falta una parte que afecta a la izquierda de la representación, al parecer perdida.

Dentro de una orla de $4,5 \mathrm{~cm}$. de anchura se aprecia, esculpida en bajorrelieve, una escena en la que hay un personaje central, seguramente una figura varonil, bifronte y sentado sobre una silla de tijera que aparece sobreelevada encima de un poyo, ya que los pies del personaje

* Universidad de Murcia. 

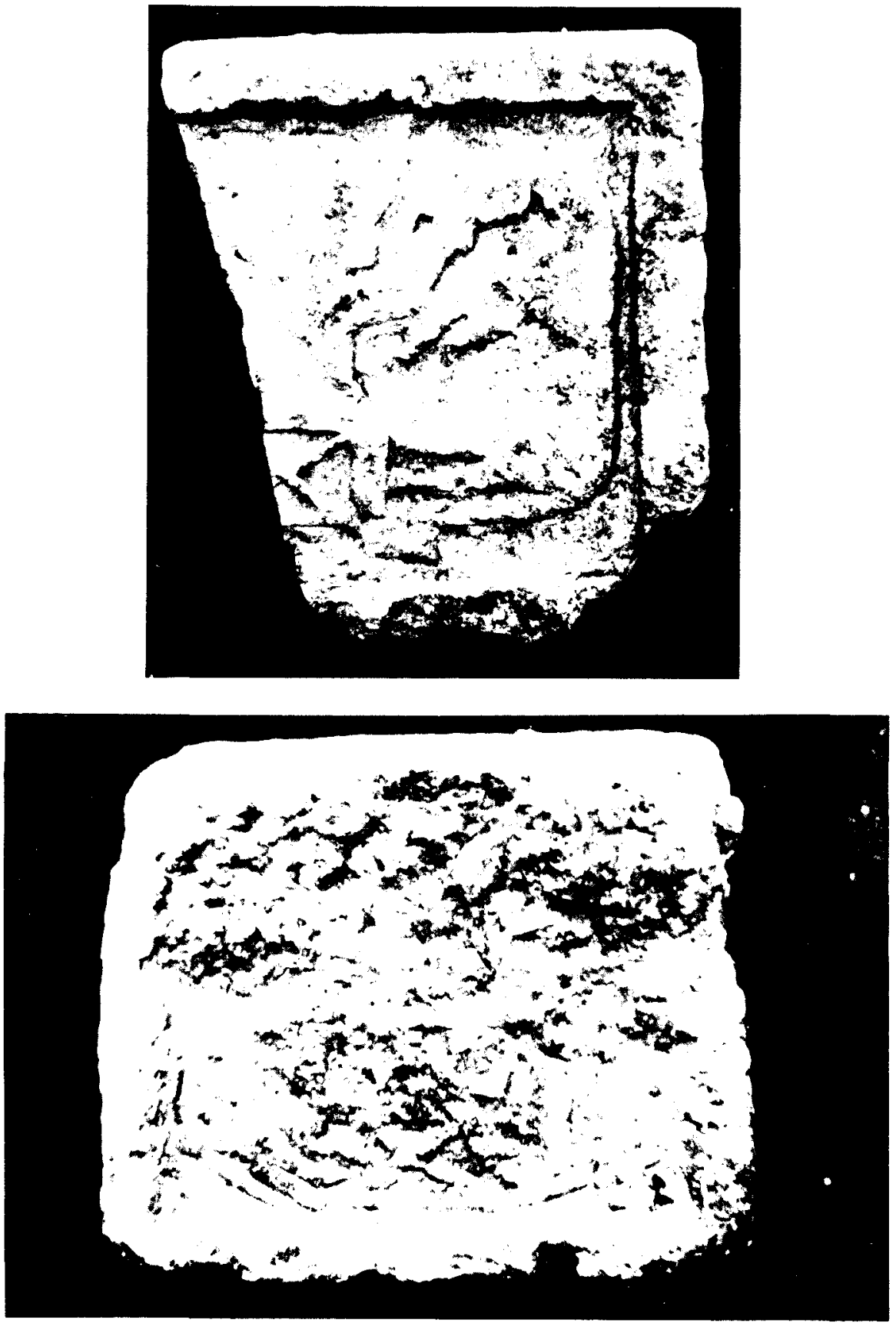

Lámina I. Los relieves de Lorca, después de su limpieza. 

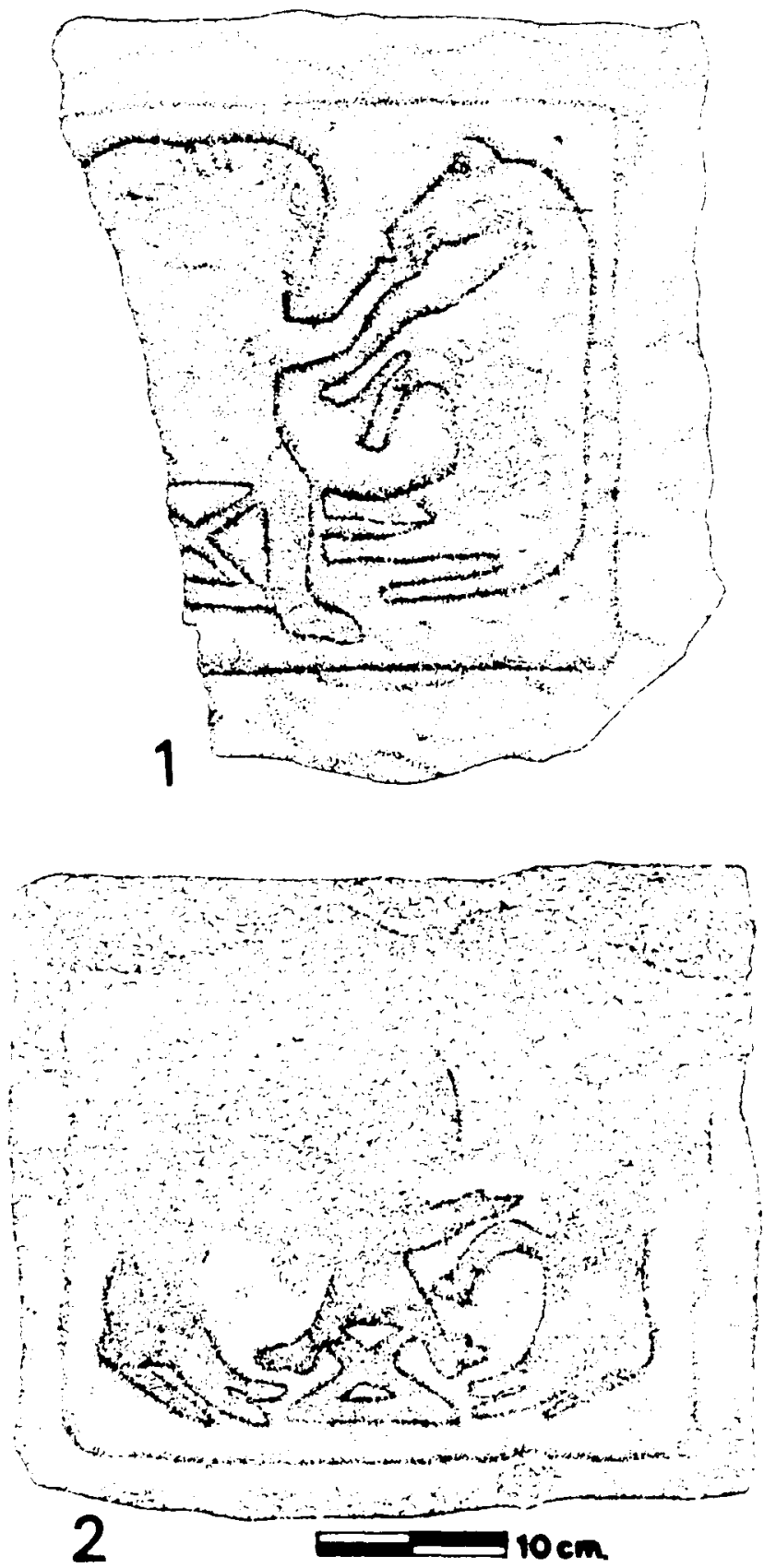

Fig. 1. Los relieves de Lorca (calco). 
se prolongan más abajo de su base. Esta figura humana extiende su mano izquierda hasta tocar el belfo de un caballo. Su brazo forma ángulo obtuso con el antebrazo. El caballo aparece apoyado sobre sus cuartos traseros, aunque la tosquedad del relieve hace que la cola aparezca más baja. Las patas delanteras están dobladas como en actitud de movimiento, rampante, sin llegar a apoyarse en el cuerpo del personaje central No se observan más detalles, como riendas, silla de montar o pretal.

Todo el relieve es plano, desarrollado entre un encuadre sobreelevado en el bloque pétreo, y está labrado con bastante tosquedad, sin llegar a tener la calidad de otros semejantes conocidos, como los de Villaricos (lám. l-1 y fig. 1-1).

RELIEVE 2. Es también un bloque de piedra caliza organógena de 40 $\mathrm{cm}$. de altura, $32 \mathrm{~cm}$. de anchura y $22 \mathrm{~cm}$. de fondo, en el que, dentro de una orla de unos $3 \mathrm{~cm}$. de anchura se aprecia, esculpida en bajorrelieve, la parte inferior de una composición en la que hay una figura humana sentada sobre una silla de tijera, seguramente bifronte, como el relieve $n .{ }^{\circ} 1$, en medio de dos caballos afrontados y rampantes que se apoyan sobre sus cuartos traseros. El personaje central parece estar tocando con sus manos los belfos de ambos animales, aunque sólo se aprecie la prolongación de su brazo izquierdo. Este personaje, a diferencia del de el bloque $n .^{\circ} 1$, parece de tamaño más reducido siendo sus posturas más forzadas sobre la silla de tijera, ya que ambas piernas aparecen abiertas en ángulo y sus pies no llegan a tocar el suelo sobre el que se apoya el asiento, sino que permanecen colgados a la altura de la intersección de las patas de la silla.

El relieve, que es también plano, está muy deteriorado en la parte superior, resultando muy difícil apreciarlo. Las figuras son de escasa calidad técnica y artística, poniéndose de manifiesto más marcadamente que en relieve $n .^{\circ} 1$ una cierta ingenuidad expresiva (lám. 1-2 y fig. 1-2).

En Murcia se conoce otro relieve más, hallado en la Encarnación, Caravaca, y descubierto en 1982 (San Nicolás, 1983-84, 2, 77). Los relieves de Lorca son del tipo sedente, mientras que el de Caravaca es del tipo estante, según la división que hizo A. Fernández de Avilés (Fernández de Avilés, A, 1942).

En la Península Ibérica hay 11 relieves similares representando al potnios hippōn y todos ellos aparecieron en el cuadrante sudoriental peninsular, la mayor parte en localidades próximas a la costa o costeras, 
entre Valencia y Almería, excepto los dos hallados más al interior de Jaén y Albacete. De estos relieves seis son del tipo estante: los dos de Sagunto y los de Mogón, Balones, Ciudadela y Caravaca. Los otros cinco son del tipo sedente: los dos de Villaricos, el del Llano de la Consolación y los dos de Lorca (fig. 2).

Los relieves de Lorca, sobre todo el $n .^{\circ} 1$, tienen su paralelismo más claro en el de Villaricos (Almería), del Museo Arqueológico de Barcelona. En este relieve, que es también plano, se representa al domador de caballos sentados en la silla de tijera, de forma bastante más realista que en los de Lorca, que son algo más esquemáticos. También los caballos del relieve de Villaricos ofrecen más detalles que los lorquinos: las crines, los belfos, algún rasgo de la musculatura, el pretal...etc., que los hacen aparecer bastante más naturalistas. El personaje masculino de Villaricos, que tiene rostro bifronte, se sienta en la silla con las piernas abiertas a un nivel superior al de la base sobre la que están ámbos caballos, al contrario que en el relieve $n$. 1 de Lorca, en el que el domador aparece en un plano inferior. No ocurre to mismo con el relieve $n$. 2, en el que domador y caballos aparecen a un mismo nivel.

No parece probable que en los relieves de Lorca el artista hubiese plasmado los minuciosos detalles que se aprecian en el de Villaricos, ya que en éstos se observa una evidente tosquedad, sobre todo en la postura de los animales y muy especialmente en las patas traseras, que los hace más esquemáticos y que los obliga a adoptar una postura forzada, muy alejada del mayor naturalismo del relieve almeriense. Blázquez apuntó la posibilidad de que utodos estos pormenores (los detalles del relieve de Villaricos) quizá estaban representados también en los restantes relieves, pero se han perdido en ellos a causa del desgaste sufrido por las superficies" (Blázquez, 1977, 293). Creemos que no es el caso de los de Lorca, en los que parece evidente la torpeza del autor en comparación con el de Villaricos. Los caballos de Lorca están más cerca del modelo del relieve de Mogón, desde el punto de vista estilístico.

Hay una diferencia más: el caballo de la izquierda de Villaricos parece llevar pretal, mientras que en los de Lorca no se aprecia ningún aparejo.

Blázquez afirma que la silla del domador de Villaricos "parece una verdadera silla de montar, como la de Liria y las que representan algunos exvotos" (Blázquez, 1977, 293), lo cual parece confirmarse en los relieves de Lorca, sobre todo en el $n .^{\circ} 2$ en el que el domador aparece "montado" en la silla de tijera, con ambas piernas colgando, en una postura ciertamente forzada, que recuerda la de un jinete sobre su ca- 

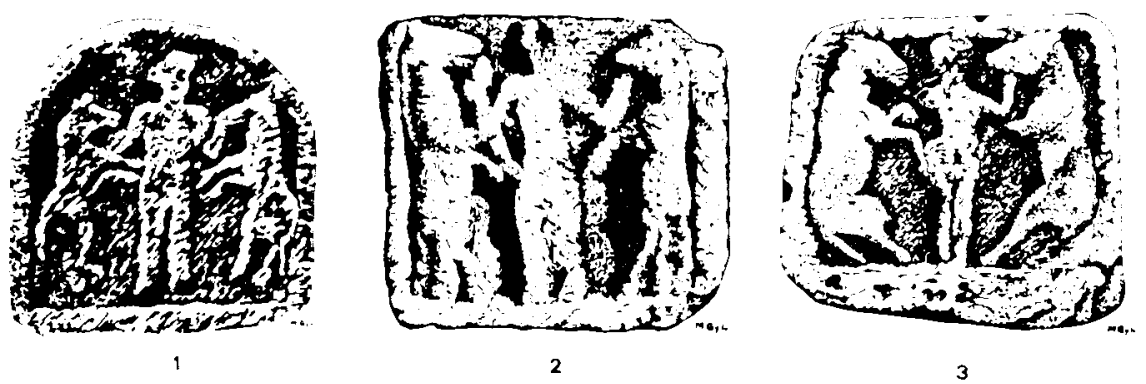

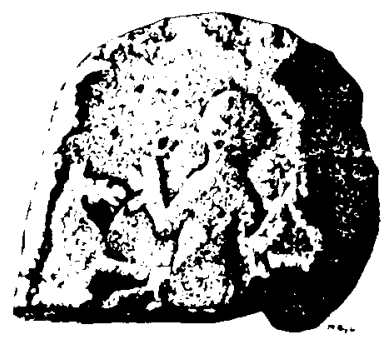

4

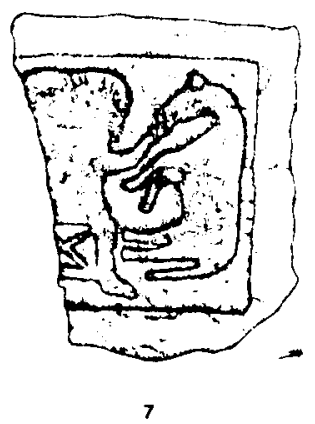

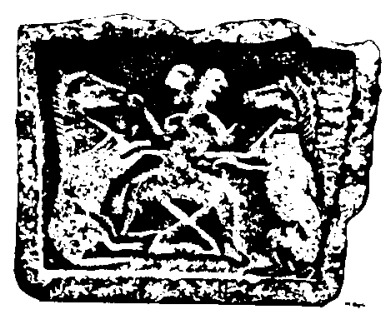

5

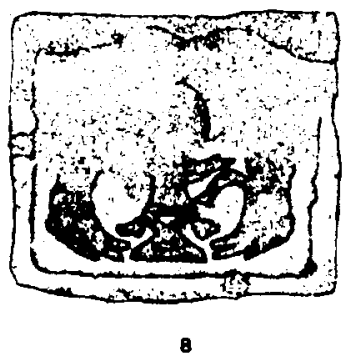

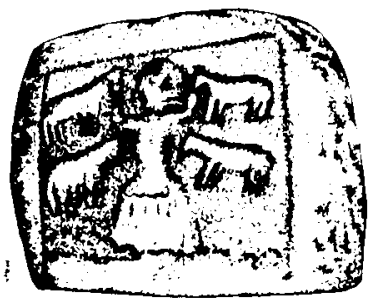

6

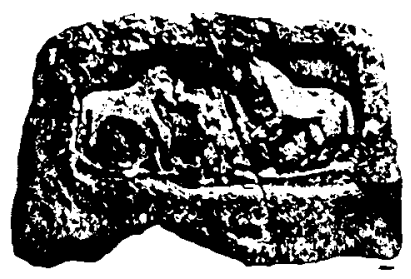

9

Fig. 2. Relieves de: 1, Sagunto; 2, Sagunto; 3, Mogón; 4, Balones; 5, Villaricos; 6 . Llano de la consolación; 7, Lorca; 8, Lorca; 9, Caravaca (1-6, según Blázquez). 
balgadura. En este relieve $n .^{\circ} 2$ es dónde mejor se aprecia ese aspecto, de entre todos los existentes.

Hemos intentado buscar una explicación para comprender el posible simbolismo de las posturas del domador y los caballos en los dos tipos de composiciones existentes: estante y sedente. En los del primer tipo, en los que el domador aparece de pie, los caballos se representan normalmente rampantes, a veces en una postura violenta o forzada. Pero en 1982 apareció el relieve de La Encarnación, de Caravaca, en el que el domador se representa estante y los caballos no se representan rampantes sino en posición normal, aunque ninguno de los dos se apoya sobre las cuatro patas sino que parecen estar iniciando un paso o brinco, ya que en ambos se aprecian las patas delanteras ligeramente elevadas. Esta misma postura parece apreciarse en los caballos del relieve del Llano de la Consolación, en el que los cuatro animales parecen tener las patas delanteras algo más elevadas que las traseras.

Es decir, que en ambos tipos de relieve hay una adecuación de la composición al personaje central, representando los caballos de manera que el domador pueda llegar, de pie o sentado, a tocar sus belfos sin adoptar posturas violentas. Para ello, en los relieves en los que el domador se representa de pie, los caballos rampantes se adaptan a la verticalidad de la figura central, incluso alterando si es preciso sus proporciones; $y$ en el tipo sedente, los animales se «sientan» también en el suelo o cambian hacia un esquema de horizontalidad que los adapta a la posición del domadoi. En todo caso, los caballos no se representan totalmente estáticos, sino en distintos grados de movimiento. $Y$ todo ello parece servir para reforzar el protagonismo del personaje central.

En cuanto al aspecto cronológico, surgen algunas dudas al intentar establecer una evolución de ambos tipos. Fernández de Avilés afirmó que los dos relieves de Villaricos y el del Llano de la Consolación, todos del tipo sedente, serían lás últimas derivaciones del tema, señalando que la procedencia de la vieja Baria indicaria una época tardía (Fernández de Avilés, A., 1942). Los dos relieves de Lorca también parecen tardios en relación con los restantes, ya que los hemos fechado a fines del III a.J.C., de acuerdo con los materiales arqueológicos que parecen asociarse a ellos. Pero el relieve de Caravaca, que es del tipo estante, se ha fechado «entre el siglo III y la romanización» (San Nicolás, 1983-84, 277), también de acuerdo con los materiales arqueológicos asociados, que no se especifican en la publicación, pero que según dato verbal del autor eran «fragmentos de paredes de cerámica común y cerámica gris, que correspondían a pequeñas ollitas, y un fragmento de cerámica pintada, tal vez ibérica". 
De acuerdo con esta circunstancia se puede deducir que parece no existir una línea evolutiva clara entre los dos tipos que nos permita fechar todos los relieves del tipo estante como anteriores a los del tipo sedente. $Y$ tampoco el aspecto artístico parece aclarar mucho en este sentido, ya que en los relieves del tipo sedente, al lado de la buena calidad del de Villaricos del Museo Arqueológico de Barcelona, están los más mediocres de Lorca y el ingenuo del Llano de la Consolación.

Sin embargo, la similitud cronológica de los dos relieves de Lorca y el de Caravaca, que pudieron ser contemporáneos, tal vez, hacia el siglo III a. J.C., nos parece un dato de interés para la cronología general de este tipo de representaciones y, como afirma Blázquez (Blázquez, $1977,305)$, nos parece que estas representaciones pueden enmarcarse entre los siglos VI y III a. J.C. aunque, a nuestro juicio, no parece hoy posible establecer un orden cronológico de aparición ni asegurar una línea evolutiva clara entre los dos tipos conocidos.

En cuanto a la interpretación, creemos que los trabajos publicados sobre el tema, últimamente los de Chapa (Chapa, T., 1984) y, sobre todo, Blázquez (Blázquez, 1977), han aclarado bastante su comprensión.

Es claro que todos estos relieves proceden de una zona que en España es la más abierta a las influencias mediterráneas de colonizadores griegos y púnicos y por ello es lógico pensar en una influencia por vía marítima de procedencia oriental, ya que en estas representaciones no parecen apreciarse influencias romanas ni el tema tiene antecedentes peninsulares que pudieran justificar su origen local.

Blázquez planteó, en 1975, la posibilidad de un orígen chipriota, significando la influencia de Chipre sobre la España prerromana. F. Benoit, que se apoyó en antecedentes cretenses, también propone un origen oriental, de Creta o de algún otro lugar del Mediterráneo donde el tema del potnios hippōn era frecuente. T. Chapa expone igualmente la posibilidad del origen mediterráneo oriental, matizando que estas manifestaciones parecen indicar la existencia de unos contactos con áreas por las que se extiende el culto a una divinidad de los animales "desde el área siria a Fenicia, Grecia, Etruria y península Ibérica" (Chapa, 1988, 184).

Estas opiniones contrastan con la cronología tardía que para estos relieves propusieron García y Bellido, ya en época romana (Garcia y Bellido, 1949) y Fernández de Avilés, que los calificó de "hispanorromanos" y los supone las últimas derivaciones del tema (Fernández de Avilés, 1950, 126). 
Aunque se manejan paralelismos orientales para las representaciones, Blázquez, después de analizar el tema del despotes theron en el ámbito etrusco, ha propuesto una relación de los ejemplares de Etruria y Villanova con los de Esparta y Rodas, así como con representaciones en vasos de figuras rojas del siglo VI a. de J.C., deduciendo de ello la cronología entre los siglos VI y III a. J.C. y descartando la influencia romana, ya que el tema parece proceder ciertamente del ámbito mediterráneo y parece seguro que el denominado por Nilsson "Master of Animals" (Nilsson, 1951,507$)$ es, en este caso, una divinidad protectora de los caballos que desapareció cuando fue sustituido por Hermes, en opinión de Chittenden, llegando el tema a la Península antes de que Hermes hubiese asumido ese carácter.

Sin embargo, ya hemos apuntado en otra ocasión que la separación entre divinidades orientales e ibéricas en el levante y sur peninsulares, y muy especialmente en zonas de influencia mediterránea directa, es bastante compleja, ya que en esa etapa prerromana se habian producido sincretismos de divinidades indígenas con divinidades clásicas, de forma que en el mejor de los casos, los restos arqueológicos, los contextos en los que éstos aparecen o la iconografía exenta, sólo nos permiten deducciones que, en gran medida, deben apoyarse en paralelismos tipológicos casi siempre externos y no siempre fiables (Eiroa, 1986, 73).

A modo de conclusiones, podemos apuntar que, aunque tenemos razonables dudas acerca de la contemporaneidad de los relieves de Lorca y los materiales arqueológicos hallados en el mismo lugar de su hallazgo, ya que la prospección sobre el terreno se ha realizado medio siglo después del descubrimiento de las dos piezas y ello no nos garantiza un conocimiento preciso del lugar desde el punto de vista arqueológico, hay en la actualidad tres relieves, los tres en Murcia, que se pueden asociar a materiales arqueológicos, con la prudencia que el caso requiere: los dos de Lorca, que se asocian a materiales de entre los siglos III a. J.C. al V d. J.C., con un momento de plenitud entre los siglos I y II d. J.C.; y el de Caravaca, con materiales de entre el siglo III a.J.C. y la romanización, si aceptamos la asociación de materiales referida por San Nicolás, para la que también tenemos algunas reservas.

De estos tres relieves, dos son del tipo sedente y el otro del estante. Por eso, y por lo anteriormente expuesto, creemos que no es posible hoy establecer una línea evolutiva, ni desde el punto de vista artístico ni desde el cronológico, entre ambos tipos, ya que los dos aparecen indistintamente al final del ciclo. 
Como marco cronológico para todos ellos se puede aceptar el propuesto por Blázquez, entre los siglos VI y III a. J.C., aunque creemos que no es conveniente descartar una pervivencia de los modelos hasta un momento algo más posterior, tal vez hasta principios del siglo II a. J.C., en que la romanización se intensifica en la zona.

Los dos relieves de Lorca, el de La Encarnación de Caravaca y el de Villaricos del Museo Arqueológico de Barcelona, pueden ser, a nuestro juicio, los más tardios del grupo, de hacia el siglo III a. J.C., aunque con la posibilidad de que perviviesen hasta el inicio de la romanización en el área del sureste peninsular.

Por último, parece cada vez más probable, y en ese sentido se han pronunciado sus más destacados tratadistas, que las representaciones procediesen del ámbito mediterráneo, dónde tienen diversos precedentes, a través de los contactos entre la costa levantina y las costas del Mediterráneo central y oriental. 


\section{BIBLIOGRAFÍA}

BLÁZQUEZ, J. M. ${ }^{\text {a }}$ 1975: Diccionario de las religiones prerromanas de Hispania, Madrid.

—, 1977: "Dioses y caballos en el mundo ibérico" en Imagen y Mito, 290. Madrid.

Chapa Brunet, T., 1984: La escultura ibérica zoomorfa, Madrid, Ministerio de Cultura, 179-185..

EIROA, J. J., 1986: «El kalathos de Elche de la Sierra (Albacete)» en Anales de Prehistoria y Arqueologia, 2, Universidad de Murcia, 7386.

EIROA, J. J. y MARTínez, A., 1988: “Noticia de dos representaciones de potnios hippōn encontradas en Lorca (Murcia)», en Anales de Prehistoria y Arqueologia, 3, Universidad de Murcia, 123-133.

FERNANDEZ DE AVILÉS, A., 1942: "Relieves hispanorromanos con representaciones ecuestres" en Archivo Español de Arqueología, XV, 199215.

Garcia y Bellido, A., 1949: Esculturas romanas de España y Portugal, C.S.I.C., (2 vols.). Madrid.

KEAY, S. J., 1984: "Late roman amphorae in the Western Mediterranean: A tipology and economic study (part I). BAR Intern. Series, 196.

NiLSSON, M. P., 1951: The Mynoan-Mycenaean Religion and its Survival in Greek Religion. Lund.

SAN NiCOLÁS, M., 1984: "Un nuevo relieve del "domador" de caballos procedentes de La Encarnación (Caravaca, Murcia)", en Pyrenae, 19-20, 277-279. Barcelona. 